Fanum

Sociológico

\section{Forum Sociológico}

Série II

$30 \mid 2017$

Internet e ativismos em saúde

\title{
Mães pela cannabis medicinal em um Brasil aterrorizado entre luzes e fantasmas
}

Mothers for medical cannabis in a Brazil terrified by lights and ghosts

Virgínia Martins Carvalho, Margarete Santos de Brito e Mário Gandra

\section{OpenEdition}

Journals

Edição electrónica

URL: https://journals.openedition.org/sociologico/1747

DOI: 10.4000/sociologico. 1747

ISSN: $2182-7427$

Editora

CICS.NOVA - Centro Interdisciplinar de Ciências Sociais da Universidade Nova de Lisboa

Refêrencia eletrónica

Virgínia Martins Carvalho, Margarete Santos de Brito e Mário Gandra, «Mães pela cannabis medicinal em um Brasil aterrorizado entre luzes e fantasmas», Forum Sociológico [Online], 30 | 2017, posto online no dia 30 décembre 2017, consultado o 28 juin 2022. URL: http://journals.openedition.org/ sociologico/1747 ; DOI: https://doi.org/10.4000/sociologico.1747 


\title{
MÃES PELA CANNABIS MEDICINAL EM UM BRASIL ATERRORIZADO ENTRE LUZES E FANTASMAS \\ MOTHERS FOR MEDICAL CANNABIS IN A BRAZIL TERRIFIED BY LIGHTS AND GHOSTS
}

\author{
Virgínia Martins Carvalho \\ Universidade Federal do Rio de Janeiro, Faculdade de Farmácia, Departamento de Análises Clínicas e Toxicológicas, \\ Laboratório de Análises Toxicológicas
}

\author{
Margarete Santos de Brito \\ APEPI - Apoio à Pesquisa e Pacientes de Cannabis Medicinal
}

Mário Gandra

Universidade Federal do Rio de Janeiro, Faculdade de Farmácia, Departamento de Análises Clínicas e Toxicológicas

\begin{abstract}
Resumo
Desde a proibição da planta Cannabis sativa L. (cannabis ou maconha), diversos estudos científicos vêm sendo publicados demonstrando tanto os riscos à saúde do uso social como as evidências que embasam o uso farmacêutico. Acompanhando a ciência, ativistas têm se organizado pleiteando a regulação do uso social à semelhança das drogas lícitas como álcool e tabaco. No entanto, nem a ciência nem o movimento ativista pela legalização puderam sozinhos impingir tamanha revolução na regulação sanitária brasileira como a que vem sendo liderada por mulheres/mães que adotaram a desobediência civil pacífica como estratégia para garantir o direito à saúde e qualidade de vida de seus filhos. Este artigo trata desse movimento feminino e materno que tem transformado a realidade com auxílio das tecnologias digitais e impulsionado instituições públicas a se voltarem ao tema.
\end{abstract}

Palavras-chave: maconha medicinal, desobediência civil, movimento de mães

\begin{abstract}
Since the prohibition of Cannabis sativa L. (marihuana), many scientific studies have been published showing the risk of the recreational use, but also evidences for pharmaceutical use. Powered by these findings, activists are getting organized to request the regulation of social use of cannabis as already happens with legal drugs like alcohol. Nevertheless, neither science nor the activist movement were able to revolutionize Brazilian drug regulation by themselves, at least not anything like what is being done by women/mothers who adopted the civil disobedience strategy as a means to defend the right to health and quality of life of their children. This text tells the story of this social movement, with great feminine and maternal characteristics, which is changing reality through digital media and pressing public institutions to discuss the medical use of cannabis.
\end{abstract}

Keywords: medical cannabis, civil disobedience, mother movement

\section{Introdução}

O Brasil tem vivido recentemente uma revolução no campo da regulação sanitária relacionada ao uso medicinal da cannabis. Essas mudanças estão ocorrendo pela reivindicação de mães de crianças portadoras de doenças neurológicas que assumiram o risco de responderem penalmente por tráfico internacional de drogas ao importar extratos de cannabis para o tratamento de epilepsia refratária ${ }^{1}$ de seus filhos e pelas ações que envolvem diversos atores como ativistas, cientistas, juristas, mídias, 
produtores de extratos de cannabis e pacientes. A mobilização desses atores foi grandemente facilitada pelo emprego das mídias sociais como ferramenta estratégica, desde grupos de WhatsApp até redes sociais como o Facebook que serviram de veículo de difusão do conhecimento e congregação de pessoas para organização de movimentos sociais.

A emergência do tema no debate público vem sendo marcada por uma polêmica entre a demanda pelo acesso à cannabis para fins medicinais e o status ilegal da planta, apesar de diversos estudos apontarem sua eficácia terapêutica e de seus derivados (Cunha et al., 1980; Hussain et al., 2015; Mechoulam et al., 2007; Whiting et al., 2015).

As propriedades medicinais da planta Cannabis sativa L. (cannabis, maconha, dirijo, marijuana, marihuana, ganja, marimba, liamba, etc.) foram descritas na farmacopeia chinesa Pen-Ts'ao Ching há 2000 anos, considerada a primeira farmacopeia conhecida do mundo (Honório et al., 2006). O Dicionário de Plantas úteis do Brasil e das Exóticas Cultivadas, de autoria de M. Pio Corrêa, publicado em 1926 (compêndio de referência na área farmacêutica), descreve as características botânicas e propriedades medicinais da Cannabis sativa L., que denominou genericamente de "cânhamo verdadeiro" (Corrêa, 1926) e a Farmacopeia Brasileira publicada em 1929 também descreve a Cannabis sativa L., var. indica, com a denominação genérica "maconha", "meconha", "diamba" e "liamba", indicando como matéria-prima as sumidades floridas na elaboração oficinal de extrato fluido de cânhamo-da-índia, pó de cânhamo-da-índia e tintura de cânhamo-da-índia (Silva, 1929). Os princípios ativos mais abundantes na planta, canabidiol (CBD), composto ansiolítico, e $\Delta$ 9-tetrahidrocanabinol ( $\triangle 9$-THC), composto euforizante, foram isolados em 1940 (Adams et al., 1940) e 1964 (Gaoni e Mechoulam, 1964), respectivamente, e amplamente estudados quanto às propriedades farmacológicas. Além desses princípios ativos, a planta contém dezenas de outros canabinoides (estruturas químicas semelhantes ao CBD e THC) e óleos essenciais conhecidos quimicamente por terpenos com potencial terapêutico (Honório et al., 2006).

Se por um lado a Convenção Única de Entorpecentes da Organização das Nações Unidas de 1961 e suas atualizações permitem o uso médico e científico de qualquer droga proibida, na prática o uso médico da cannabis foi ao longo dos anos pós-proibição sendo impedido pela regulação sanitária.

A busca pelo tratamento com cannabis no Brasil foi impulsionada pela informação através da internet sobre o sucesso da terapia no controle de convulsões em crianças com epilepsia refratária.

A epilepsia é um distúrbio cerebral crônico caracterizado pela ocorrência periódica e imprevisível de convulsões. As crises são causadas por descargas elétricas anormais dos neurônios cerebrais que podem ocorrer em qualquer idade. Não se sabe ao certo a causa que inicia exatamente a crise convulsiva e o que leva o seu encerramento (McNamara, 2012). Estudos estimam que a prevalência mundial de epilepsia ativa seja de 0,5\% a $1,0 \%$ aproximadamente e que $30 \%$ desses pacientes sejam refratários, ou seja, continuam a ter crises mesmo com tratamento (Kwan e Brodie, 2000) e foi justamente em um caso de epilepsia refratária que o extrato de cannabis rico em CBD se mostrou eficaz.

Uma experiência internacional emblemática que influenciou as famílias a buscarem essa via terapêutica foi o caso da menina britânica Charlotte Figi, que vinha sofrendo vários episódios de crise convulsiva desde os primeiros meses de vida. Foi diagnosticada com síndrome de Dravet aos 2,5 anos, ocasião em que apresentava inúmeras convulsões diárias que resultaram na perda das habilidades de andar, falar e comer. Sua mãe e seu pai, inconformados com a ineficácia dos tratamentos tradicionais e com a incapacidade dos profissionais médicos de lidarem com a questão, começaram a estudar por conta própria tratamentos alternativos até iniciar no Colorado (EUA) uma terapia com cannabis que controlou as crises convulsivas na primeira semana de tratamento com retorno gradativo das habilidades neuromotoras (Blakely, 2014). Diante desta experiência, mostrada em documentário lançado em 2013 pela CNN através de uma série de reportagens chamada Weed, dirigida por Sanjay Gupta e por informações na rede social Facebook, mães e pais de crianças portadoras de epilepsias refratárias de várias partes do mundo começaram a buscar o tratamento, estimulando médicos a estudar o assunto e considerar a terapia com cannabis, principalmente porque nos EUA o extrato de cannabis da variedade cânhamo, isto é, rico em CBD, já era legalmente comercializado como suplemento alimentar. Assim, algumas famílias encontrando apoio em um reduzido número de médicos e outras por conta própria enfrentaram a proibição, passando a importar tais extratos ou obtendo-os pelo apoio de redes secretas de cultivadores que produziam esses extratos regionalmente.

Embora em países vizinhos como Colômbia e Argentina já houvesse a aceitação do uso pessoal e o Uruguai já estivesse se encaminhando para a legalização da cannabis em 2012 (Carvalho e Martínez, 2014), no Brasil imperou a inércia das comunidades médica e farmacêutica diante das evidências científicas e clínicas, até que famílias tendo conhecimento do sucesso do tratamento nos EUA, através das redes sociais, começaram a importar extratos de cannabis para tratar as crises convulsivas de seus filhos. Neste cenário, mães e pais se organizaram coletivamente articulando com (1) alguns médicos que aceitaram acompanhar tal tratamento e (2) 
com movimentos ativistas pela legalização do uso terapêutico e recreativo da cannabis para pressionar as instituições. A situação dessas famílias e de outros pacientes foi retratada no documentário ILEGAL, a vida não espera, lançado em outubro de 2014, dirigido por Raphael Erichsen e Tarso Araújo, que mostraram a luta de 5 mulheres, com diferentes problemas, todas utilizando ilegalmente cannabis e seus derivados como remédio, e os trâmites políticos relacionados à permissão do uso da maconha para fins medicinais. Sequencialmente, a divulgação da utilização dos extratos de cannabis ricos em CBD com bons resultados em crianças com epilepsia refratária e doenças raras foi amplamente noticiada pela mídia nacional e internacional, aumentando rápida e significativamente o número de pacientes que optaram por esse tratamento. Porém, de acordo com a legislação brasileira vigente na época, o fato do CBD ser derivado da maconha configurava a importação dos extratos como tráfico internacional de drogas.

Este trabalho faz uma narrativa da observação do fenômeno e análise do contexto do tratamento de saúde com cannabis que vem revolucionando a regulação sanitária no Brasil. Esse fenômeno é caracterizado pela mobilização social que emprega o ciberativismo como ferramenta para garantia do direito à saúde e do direito individual do paciente e de seu responsável legal em decidir sobre seu próprio tratamento.

Essa observação e análise do fenômeno mostram a grande capacidade de transformação da realidade empregando os recursos cibernéticos que possibilitam rápida divulgação de imagens, informações e ideias capazes de congregar um grande número de pessoas para pressionar os sistemas regulatório e jurídico para garantir seus direitos.

\section{Narrativa}

O controle de plantas e substâncias previstas na Convenção Internacional de Drogas é regulada no Brasil através da Portaria n. ${ }^{\circ} 344$, de 12 de maio de 1998, que aprova o regulamento técnico sobre substâncias e medicamentos sujeitos a controle especial (Brasil, 1998), sendo esta regulamentação aplicada e fiscalizada pela Agência Nacional de Vigilância Sanitária (Anvisa). Esta portaria complementa a Lei 11.343/06 (Lei de Drogas), sendo que a importação de todas as plantas e substâncias alocadas nas listas E e F, respectivamente, constitui tráfico internacional de drogas, exceto quando se tratar de material certificado para medições laboratoriais ou para pesquisa atendendo à regulamentação específica normatizada atualmente pela RDC2 55/2013 (Brasil, 2013a). A despeito das evidências da eficácia terapêutica dos compostos naturais (fitocanabinoides) da cannabis, os mesmos não receberam a mesma lógica farmacêutica dos compostos do ópio, cujo fármaco mais conhecido é a morfina, amplamente utilizada no controle da dor. Assim, importar extratos ricos em CBD só foi possível legalmente a partir de 2015, quando a Anvisa autorizou em caráter excepcional, após o Conselho Federal de Medicina (CFM) aprovar através de norma específica a prescrição de CBD mediante pressão de movimentos sociais e da mídia. Após o lançamento do filme ILEGAL, a vida não espera ocorrido em outubro de 2014, o CFM publicou em dezembro a Resolução N. 2.113/2014, permitindo aos médicos de todo o Brasil - das especialidades de Neurologia, Psiquiatria e Neurocirurgia - prescreverem compassivamente o CBD para pacientes portadores de epilepsia até 18 anos (Brasil, 2014). Na sequência, adequando a regulação sanitária à normativa do CFM, a Anvisa atualizou, em 2015, a lista de substâncias controladas através da RDC n. ${ }^{\circ}$ 03/2015, que incluiu o canabidiol (CBD) na lista C1, mesma classificação de medicamentos como o ácido valproico, carbamazepina e olanzapina, por exemplo (Brasil, 2015a). A partir desta atualização, a Anvisa emitiu a RDC n.0 17/2015 e autorizou a importação de produtos à base de CBD para uso terapêutico em caráter excepcional, ocasião em que permitiu apenas produtos que apresentassem teor de canabidiol superior ao de THC (Brasil, 2015b). Um ano depois, em cumprimento à decisão judicial proferida, em 03 de março de 2016, nos autos da Ação Civil Pública n. ${ }^{0}$ 0090670-16.2014.4.01.3400 (Ministério Público Federal, 2016), a Anvisa publicou, em 21 de março de 2016, a RDC n. ${ }^{0}$ 66/2016, permitindo a importação para uso médico pessoal da planta, partes da planta e de seus compostos, inclusive o THC, desde que os mesmos apresentem registro sanitário (Brasil, 2016).

A cronologia da regulação recente trata de uma conquista altamente significativa que deve ser atribuída, principalmente, às mães das crianças epiléticas que tiveram suas histórias e pleitos veiculados na mídia impressa, televisiva e internet. Essas mães vêm ocupando a liderança da cannabis medicinal no Brasil, se articulando com movimentos ativistas, instituições de ensino e pesquisa, levando a demanda de democratização do acesso que impulsiona o desenvolvimento de pesquisas (figura 1 ).

Um exemplo de conquista protagonizada por essas mães é o projeto Farmacannabis da Universidade Federal do Rio Janeiro (UFRJ), que vem dando apoio farmacêutico integral aos pacientes em terapia com cannabis e que foi criado pela vivência junto às associações Abracannabis (Associação Brasileira para Cannabis) e Apepi (Associação de Apoio à Pesquisa e Pacientes de Cannabis Medicinal), que juntas vinham fazendo um trabalho de apoio às famílias de pacientes portadores de enfermidades neurológicas. Enquanto a Abracannabis ensinava vários pacientes a cultivar e preparar extratos de 
Figura $1 \triangleright$ Foto da marcha da maconha e de famílias em protesto na ANVISA pelo cancelamento da audiência pública em que seria discutida a reclassificação do CBD na portaria 344/98 em 2014

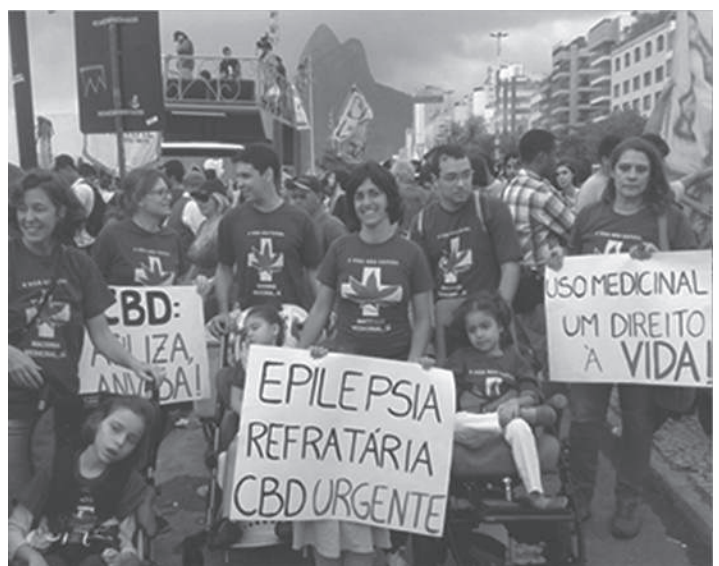

Fonte: Arquivo pessoal de Margarete Santos de Brito.

cannabis e dava apoio jurídico, a Apepi liderou a campanha publicitária do financiamento coletivo criado junto com a UFRJ para estruturar o laboratório que, por sua vez, vinha monitorando a preparação dos extratos medicinais, e que atualmente analisa os extratos empregados na terapia.

A campanha de arrecadação de fundos para o projeto Farmacannabis foi focada nas redes sociais, principalmente no Facebook, onde foram divulgadas

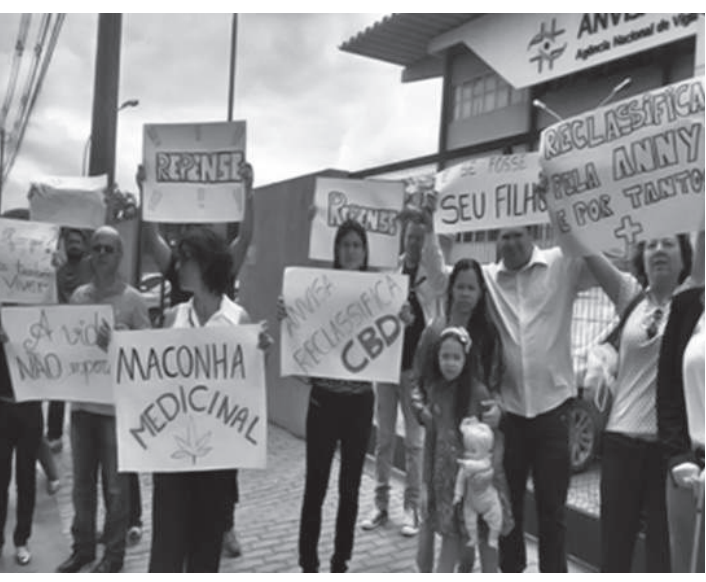

imagens de pacientes beneficiados pelo projeto e ações presenciais em eventos públicos e feiras livres, que resultaram na arrecadação de valor que ultrapassou a meta em $133 \%$, com 815 apoiadores (figura 2). Uma imagem veiculada na fanpage da Apepi teve mais de 8900 curtidas, 77 mil compartilhamentos e um alcance de mais de 9 milhões de pessoas (figura 3), fazendo a página da Apepi sair de 6 mil para 34 mil seguidores.

Figura $2 \triangleright$ Fotos da campanha de arrecadação de fundos para o projeto Farmacannabis, postado na fanpage da Apepi com alcance de mais de 12 mil pessoas, e a página virtual do financiamento coletivo
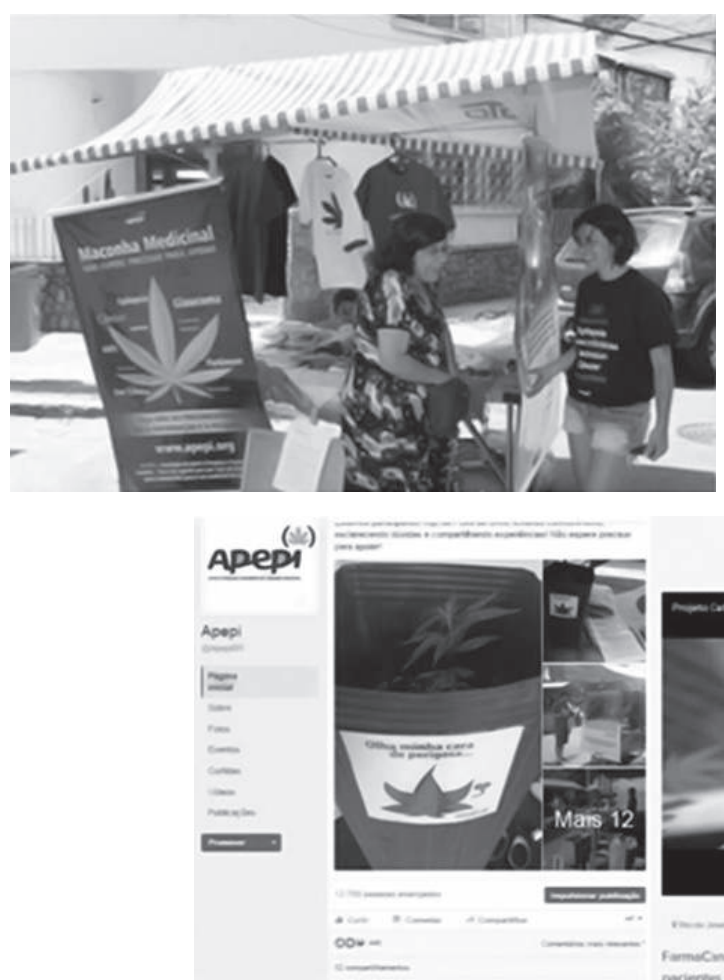

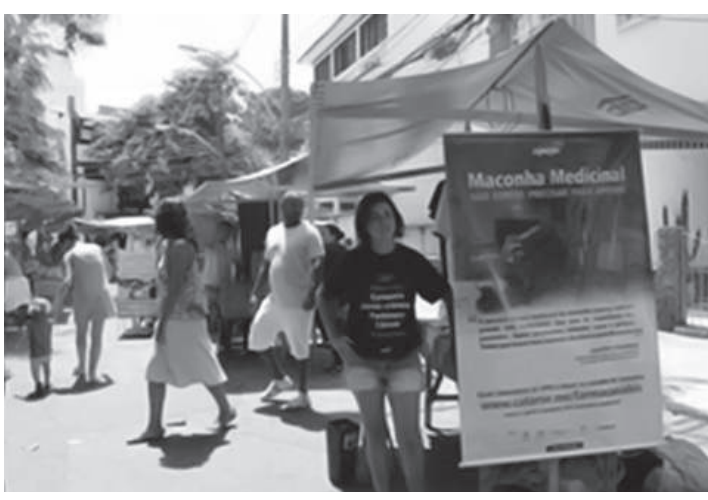

SOS cannabis medicinal

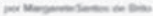

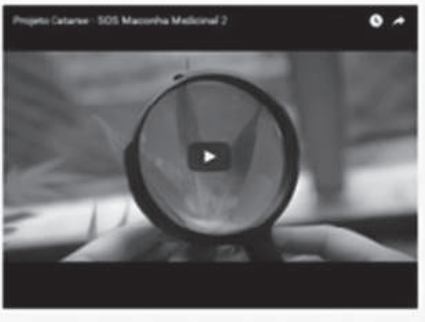

R\$ 80.767

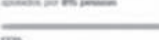

g $\cos n \operatorname{sen} 0$

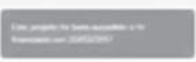

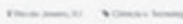

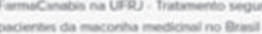


Figura 3 Imagem veiculada na fanpage da Apepi, no dia 6 de janeiro de 2017 para a divulgação do projeto Farmacannabis
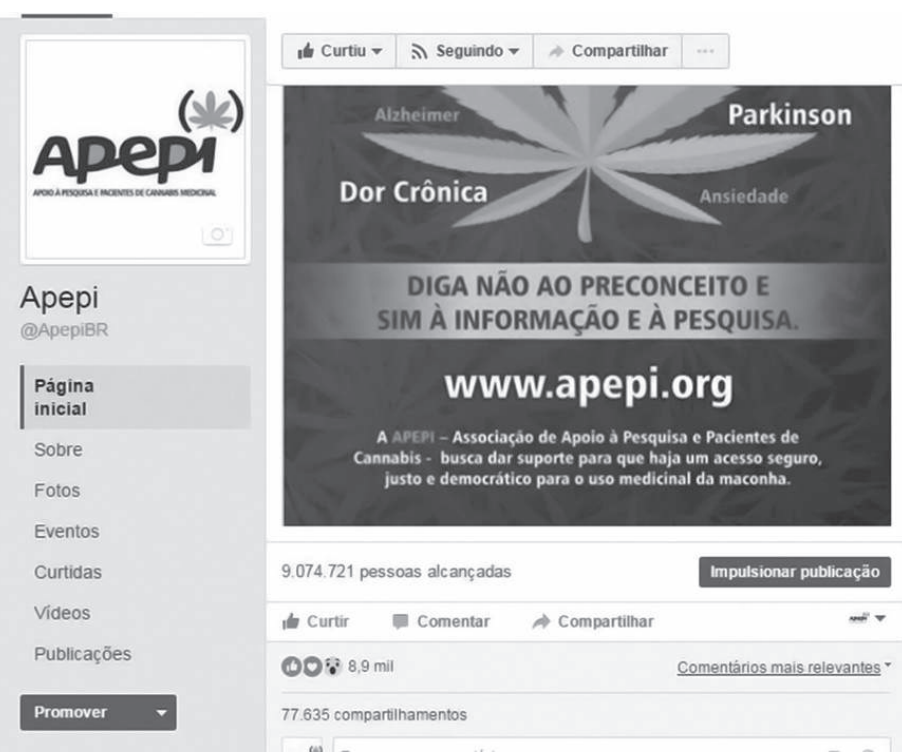

\section{Análise do contexto}

Apesar do suposto avanço na regulação do uso medicinal da cannabis, o tratamento ainda é considerado experimental, não tendo ampla aceitação médica e considerado como um recurso restrito ao uso compassivo, isto é, em casos em que todos os medicamentos registrados no país se mostraram ineficazes.

De um lado temos pacientes portadores de dor crônica ou outras desordens graves (Parkinson, esclerose múltipla, câncer sob quimioterapia, Huntington, Alzheimer, etc.) e responsáveis por crianças epiléticas e autistas com episódios de autoagressão que enxergam o uso terapêutico da cannabis como última alternativa para suas condições de saúde e, de outro lado, médicos sem conhecimento sobre os efeitos terapêuticos da cannabis, seja por falta de pesquisa, seja por

O projeto e a arrecadação de fundos foram sustentados pela necessidade das famílias em saber a segurança e melhorar a terapia de seus filhos, uma vez que os extratos importados não são produtos farmacêuticos, mas suplementos alimentares, e principalmente pela necessidade de apoio em relação ao cultivo e produção de extrato por quem não tem condições de importar, restando como única opção a desobediência civil baseada no "Estado de Necessidade de Preservação da Vida". Essa desobediência foi mostrada na rede social Facebook por uma família antes de conseguir o primeiro salvo-conduto judicial no Brasil para cultivar cannabis e o post com a foto do cultivo na varanda da família teve mais de 7500 curtidas e 3500 compartilhamentos. desinteresse oriundo de preconceitos com o status ilícito da planta e, ainda, por temer processos ético-disciplinares. Em geral, médicos temerosos de receitar derivados de cannabis desconhecem a Resolução RDC n.o 38, de 12 de agosto de 2013 - que aprova o uso compassivo de medicamento ainda sem registro na Anvisa que esteja em processo de desenvolvimento clínico, destinado a pacientes portadores de doenças debilitantes graves e/ou que ameacem a vida e sem alternativa terapêutica satisfatória com produtos registrados no país (Brasil, 2013b). Esse desconhecimento e inércia gerada pelo medo contribuíram para um cenário onde mães de crianças epiléticas realizam manobras ilegais para ter acesso ao extrato de cannabis na esperança de manter a vida de seus filhos.

Ao utilizar o termo "canabidiol", o CFM demonstrou o desconhecimento da matéria, indicando que a resolução foi mais impulsionada pela pressão social do que pelo conhecimento da terapia em curso, isto porque, neste momento, os produtos autorizados pela Anvisa tratavam de extratos de cannabis ricos em CBD, mas não do fármaco isolado. Por sua vez a Anvisa, mesmo tendo autorizado o dronabinol (que se trata do THC isolado de nome comercial Marinol ${ }^{\circ}$ com estrutura química idêntica ao THC natural da planta e com iguais propriedades fármaco-toxicológicas), alocando-o na lista A3 da Portaria de 344/98, através da Resolução n. ${ }^{\circ} 98$ de 22 de novembro de 2000 (Brasil, 
2000), manteve o THC da planta na lista de substâncias proscritas (Lista $F$ ), até que em março de 2016 uma decisão judicial resultou na RDC 66/16, em que a Anvisa permitiu a importação da planta Cannabis sativa L., de partes da planta e do THC desde que os mesmos tivessem registro sanitário (Brasil, 2016). Curiosamente o único medicamento registrado no país atualmente é o Mevatyl (registrado sob o nome Sativex $\AA$ no Reino Unido pela GW Pharma), que se apresenta na forma de extrato hidroalcoólico cujo registro foi solicitado em 2014 e que apresenta teor de THC superior ao de CBD, não sendo indicado para o tratamento da epilepsia nem para menores de 18 anos (Brasil, 2017).

É fato que a reclassificação do CBD pela Anvisa em 2015 retirou da ilegalidade algumas famílias que importavam clandestinamente o produto. Porém, devido à inexistência de qualquer extrato de cannabis rico em CBD com registro no país, a importação continuou a ser feita através da excepcionalidade e rígido controle, permanecendo a grande dificuldade para importação. No decorrer do ano de 2015, notou-se que a importação não garantia o amplo acesso ao medicamento, seja pelo alto custo, seja pela falta de médicos em prescrever e fornecer os documentos necessários para a autorização junto à Anvisa, continuando os pacientes num grande vazio entre o direito ao tratamento de saúde e a marginalidade.

No ano de 2015, a epilepsia foi classificada como um problema de saúde pública pela Organização Mundial da Saúde, Organização Pan-Americana de Saúde e Ministério da Saúde. Embora os dados epidemiológicos sejam escassos pelo fato da epilepsia não ser uma enfermidade de notificação compulsória, estima-se que sejam diagnosticados no Brasil, a cada ano, 340 mil novos casos, com a existência de 1,8 milhão de pacientes com epilepsia ativa, sendo elevada em crianças portadoras de retardo mental, paralisia cerebral, autismo e outros distúrbios de comportamento. No caso de autismo, aproximadamente $30 \%$ das crianças podem apresentar diferentes tipos de crises epilépticas durante a adolescência (Maranhão et al., 2011). As epilepsias refratárias correspondem a $20 \%$ dos casos (Alvarenga et al., 2007) e neste cenário é crescente o número de indivíduos que buscam tratamento com cannabis - quem tem condições financeiras importa os extratos com custo entre US\$ 150 e US\$ 400 por frasco contendo $30 \mathrm{ml}$ do produto, sendo que o custo mensal do tratamento pode chegar a US\$ 5000, dependendo da idade, peso e dose necessária.

Apesar do CBD ter sido liberado para uso controlado em caráter excepcional, não há disponibilidade de acesso à medicação no país, contrariando os princípios norteadores da Universalidade e da Equidade do Sistema Único de Saúde (SUS) no Brasil. A falta de acesso às necessidades de saúde faz com que as pessoas tenham que recorrer ao mercado internacional, quando é dever do Estado brasileiro a garantia das mesmas. Alguns pacientes ingressaram com ações judiciais, para que o tratamento seja custeado pelo Estado, porém a demora e a dificuldade em executar as decisões junto às secretarias estaduais ou municipais (quando a decisão é favorável) fazem com que muitos pacientes desistam e procurem os mercados ilegais.

Gera-se um problema de grande dimensão porque, além da situação de ilegalidade, a ausência do Estado provoca o uso indiscriminado de extratos de cannabis feitos a partir de plantas desconhecidas, muitas vezes oriundas do tráfico (conhecida por maconha prensada), que pode conter inúmeros compostos neurotóxicos como altos teores de metais pesados e praguicidas, além de adulterantes com maior potencial de abuso que possam ser adicionados intencionalmente na droga como, por exemplo, cocaína e nicotina (Nascimento et al., 2015) e altos teores de THC buscados no contexto de uso social/ /recreativo (EISohly et al., 2016).

Outra consequência da falta de regulação que permita o acesso terapêutico à cannabis é a situação de iniquidade entre aqueles que dispõem de recursos para comprar o medicamento em países estrangeiros e aqueles que não dispõem. A situação é inadmissível, sobretudo se for considerado o baixo custo da produção da cannabis e de seus extratos em comparação com outros medicamentos sintéticos. Assim, o Estado, ao invés de proteger a saúde da população e dos indivíduos, coloca em risco a saúde e a integridade das pessoas que fazem uso da substância sem controle de qualidade e que recorrem ao mercado ilegal. Estas pessoas já estão em estado de vulnerabilidade por seus quadros patológicos e o Estado, ao não cumprir com o seu papel, coloca esses pacientes num estado ainda maior de vulnerabilidade.

As mães, estimuladas pelo sucesso do tratamento de seus filhos, em conjunto com outras mães que buscam solução para o grave estado de saúde de seus filhos, foram capazes de mobilizar instituições de ensino, pesquisa e opinião pública através da comunicação ativa em grupos de WhatsApp e o ciberativismo engendrado na rede social Facebook e articulação com a mídia. Essa organização por grupos de WhatsApp e o ciberativismo foram ferramentas facilitadoras para os inúmeros encontros, manifestações e atos públicos feitos presencialmente ao longo desses anos por essas famílias, liderados por mulheres e mães de crianças com epilepsia refratária (figura 4).

A universidade, como representante suprema da ciência, tem a missão de observar a realidade e contribuir para o progresso do ser humano de forma comprometida com o bem-estar social. No caso do uso terapêutico da cannabis, é necessário realizar 
Figura 5 Foto da Marcha da Maconha (maio de 2016) que mostra o Movimento liderado por mães de pacientes portadores de epilepsia refratária

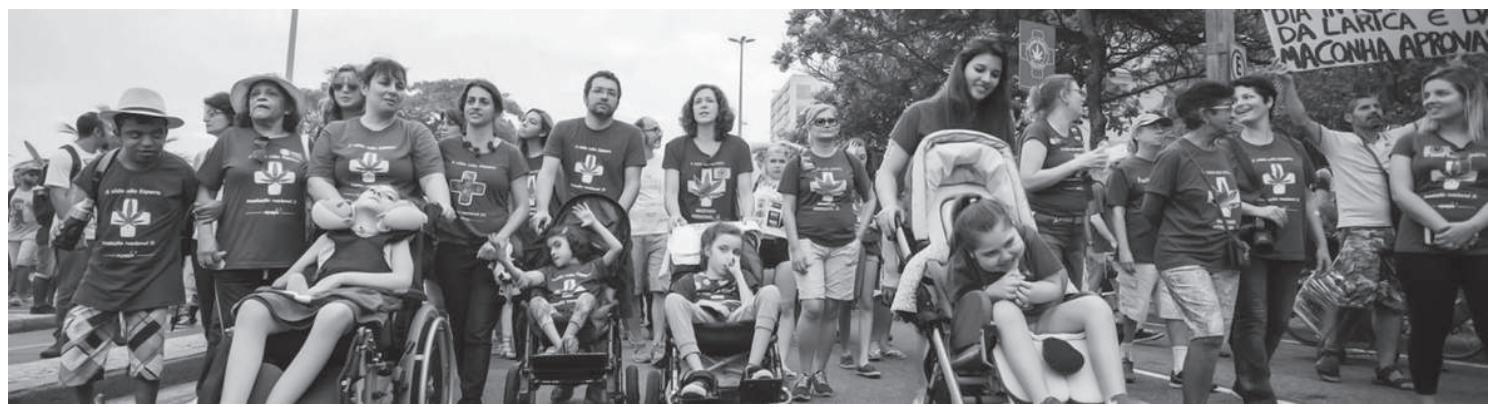

Fonte: Arquivo pessoal de Margarete Santos de Brito.

um resgate histórico de anos de inércia. É fato que o tema vem sendo estudado na Universidade Federal São Paulo (Unifesp) desde a década de 70 pelo grupo liderado pelo professor Elisaldo Carlini, que trabalhou em colaboração com o professor Raphael Mechoulam da Universidade Hebraica, sediada em Israel. Vários trabalhos foram publicados com a participação da Unifesp (antiga Escola Paulista de Medicina) (Karniol e Carlini, 1972; Karniol e Carlini, 1978; Karniol et al., 1974; Takahashi et al., 1977) e em 1980 foi publicado o primeiro estudo clínico que demonstrou a eficácia do CBD no controle das convulsões com mínimos efeitos colaterais (Cunha et al., 1980). Desde então, tanto a Unifesp quanto a Universidade de São Paulo (USP), campus Ribeirão Preto, através de pesquisadores que deram seguimento aos estudos do prof. Elisaldo Carlini, vêm publicando diversos estudos demonstrando o potencial do CBD no tratamento de várias enfermidades, sendo que recentemente a USP anunciou a inauguração do Centro de Pesquisas em Canabinoides da Faculdade de Medicina de Ribeirão Preto (FMRP) - prevista para este ano (2017) - como resultado de uma parceria com a indústria farmacêutica Prati-Donaduzzi. Mas se, por um lado, as universidades paulistas têm produzido inúmeros artigos científicos de impacto sobre o tema, pouco (ou nenhum) impacto teve na regulação sanitária da cannabis e seus princípios ativos, na postura dos Conselhos Médicos e no alívio do sofrimento e melhoramento da realidade social de inúmeros pacientes que só vieram descobrir os efeitos anticonvulsivantes através da experiência de outras famílias que desafiaram o statu quo em nome do direito à vida de seus filhos.

O cenário descrito constitui um estímulo na reflexão sobre como o que vem sendo feito no Brasil e seu impacto nas questões de saúde e como a sociedade vem se organizando e utilizando o ciberativismo na conquista do direito à saúde e a garantia de respeito à sua soberania em decidir seus modos de viver e morrer. O Sistema Único de Saúde (SUS) preconiza que a formação dos profissionais de saúde deva ser pautada na atenção integral à saúde, priorizando a atenção humanizada. No entanto, atualmente são observadas várias práticas arraigadas no processo de educação que reduzem a "atenção" a um processo mecanizado, centrado na observação de sistemas orgânicos, utilização de equipamentos e protocolos terapêuticos. Paradigmas que reforçam essa atuação mecanizada foram construídos na educação de profissionais ao longo da história, muitos dos quais estão relacionados à expansão populacional, à industrialização e ao avanço tecnológico. Nessa construção, observa-se uma separação entre o conhecimento científico e as humanidades e, além desse distanciamento de saberes, os profissionais são formados com estímulos principalmente expositivos, polarizados e extremamente setorizados, sem a integração dos saberes, o que reforça um comportamento passivo (Ceccim e Ferla, 2009; Ceccim e Feuerwerker, 2004; Serres, 1993). Provavelmente, parte dessa formação biotecnicista é atribuída à falta de experiência dos professores e pesquisadores em vislumbrar a aplicação no contexto humano e cotidiano de seu trabalho. Jorge Larrosa Bondía (2002) descreve que experiência é o que nos passa, o que nos acontece, o que nos toca. A ideia de que a experiência é o que nos acontece é distante do estar informado do que se passa no mundo, neste sentido dir-se-ia que tudo o que se passa está organizado para que nada nos aconteça. $O$ excesso de informação do que se passa no mundo é contrário à experiência, ao passo que a informação não deixa lugar para a experiência, assim o saber da informação seria o "estar informado" e a sabedoria se daria na experiência (Bondía, 2002). Esta ideia poderia explicar porque muitos pesquisadores estariam mais preocupados em publicar artigos, informar, do que desenvolver trabalhos que tenham impacto direto nas necessidades humanas.

No caso da cannabis, existe um excesso de informação empírica de quem a utiliza no contexto social/recreativo, mas pouca informação científica sobre o tratamento terapêutico com extratos e 
em pacientes pediátricos. A grande dúvida entre a comunidade médica e científica é a segurança em longo prazo relacionada à toxicidade. A contar pelo histórico da cannabis medicinal no Brasil, uma grande parcela dos profissionais de saúde ignora ao avaliar um tratamento farmacoterapêutico que todas as substâncias apresentam algum grau de toxicidade, o que diferencia o efeito terapêutico do tóxico é a condição de exposição (dose, via de administração, estado patológico, etc.). Para todos os tratamentos com fármacos o risco é uma medida probabilística calculada pelos fatores exposição x toxicidade. Assim, estudar populações que utilizam a cannabis como droga de rua em que não se sabe sua composição impede o estabelecimento adequado da toxicidade, assim como a condição de uso abusivo pela via fumada impede a extrapolação para a condição do uso medicinal por via oral.

A falta de informação científica das condições de uso medicinal no Brasil também se deve à proibição da planta e seus derivados. Uma vez que a própria Anvisa exibe uma regulação equivocada, evidenciada pela inclusão do dronabinol na lista A3 no ano 2000, enquanto manteve a mesma substância (THC) na lista $\mathrm{F}$ de proscritos até início de 2016, é previsível que os pesquisadores, ao decidirem estudar esse tema, teriam que enfrentar um tortuoso caminho burocrático pouco claro até mesmo para a agência regulatória. Assim, inúmeros trabalhos relacionados à elucidação dos mecanismos fisiológicos do sistema endocanabinoide que não requer autorizações especiais foram publicados no mundo, mas trabalhos que envolvem a manipulação de cannabis e seus compostos ficaram restritos à área forense pela facilidade dos órgãos de perícias em dispor das amostras.

O cenário atual do uso medicinal da cannabis no Brasil pode ser comparado à Alegoria da Caverna de Platão: passamos anos acorrentados pela proibição no interior de uma caverna escura de costas para a saída e quando a luz entrava, luz representada pelos estudos científicos iniciados pela Universidade Hebraica e Unifesp, pelas experiências positivas e negativas de usuários sociais/recreativos, pela experiência medicinal de comunidades tradicionais como indígenas e quilombolas e pela percepção de baixo risco pelos que utilizam a erva em visitas à Holanda ou Califórnia/EUA, essa luz mostrava não a realidade, mas sombras como fantasmas em uma visão alucinógena em que realidade se confunde com a fantasia - em certo momento alguns acorrentados, especialmente algumas mães guiadas pelo instinto de defesa da prole, conseguiram romper algumas correntes e estimularam alguns médicos e outros profissionais a segui-las na busca da luz, essas mães viraram o olhar para onde entrava a luz, desconstruíram alguns fantasmas salvando a vida de seus filhos, conseguiram estimular diversos atores sociais a olhar para a luz, mas elas e diversos pacientes ainda não chegaram à superfície. Precisam da ajuda de mais médicos, farmacêuticos, enfermeiros, professores, pesquisadores, advogados, enfim, de toda sociedade que exerce algum poder de transformação social para chegar à superfície e enxergar a luz solar, essa luz solar será o domínio no planejamento dos tratamentos com cannabis abrangendo todas as especialidades farmacêuticas possíveis por diferentes vias de administração e todo seu potencial considerando a infinidade de subsespécies e variedades de planta do gênero Cannabis. Estamos longe de sair da caverna, pode ser que jamais cheguemos a sair dela, mas o ser humano por necessidade instintiva sempre buscará a luz.

\section{Conclusão}

O movimento de mães pelo acesso democrático ao tratamento medicamentoso, apoiado por ativistas que lutam pela legalização total do uso de cannabis, tanto recreativo quanto medicinal, teve impacto decisivo na transformação da opinião pública. Os ativistas pelo uso recreativo, que já tinham experiência empírica no cultivo, garantiram o acesso de famílias a plantas e extratos artesanais e passaram a ser apoiados por mães que se juntaram ao Movimento Marcha da Maconha. Esse fenômeno, que vem sendo monitorado por instituições de pesquisa, só foi possível devido à capacidade de divulgar um grande número de informações como áudios, escritas e imagens em curto período de tempo através da internet, principalmente redes sociais (Facebook) e grupos como WhatsApp e Telegram. Esse movimento envolvendo casos de grande apelo emocional, sendo apoiado por instituições públicas reconhecidas como UFRJ, através do Farmacannabis, e Fundação Oswaldo Cruz (Fiocruz), através do grupo de trabalho Fiocannabis, que se configurou para planejar ações para atender a essa demanda de saúde, provavelmente despertou o interesse das grandes mídias, que passaram a dar cobertura em veículos de comunicação de massa (televisão e jornais de grande circulação), assim como o interesse de outras instituições de pesquisa que viram grande potencial em projetos relacionados à cannabis e empresas internacionais que comercializam extratos com capacidade financeira de patrocinar eventos de educação médica.

Os movimentos sociais em parceria com a universidade, pautados na desobediência civil pacífica exercida pelas mães, empregaram o ciberativismo como estratégia para transpor as barreiras do preconceito e convencer a sociedade brasileira que independente da discussão do uso recreativo, o acesso ao uso medicinal é urgente e legítimo. 


\section{Notas}

1 Enfermidade caracterizada por ocorrência imprevisível de convulsões que são de difícil controle.

2 Resolução de Diretoria Colegiada - Regulamentação aprovada por comissão (Diretoria Colegiada) designada pela Anvisa com anuência do Senado Federal.

\section{Referências bibliográficas}

ADAMS, R.; M. Hunt e J. H. Clark (1940), "Structure of Cannabidiol, a Product Isolated from the Marihuana Extract of Minnesota Wild Hemp", Journal of the American Chemical Society, 62 (1), pp. 196-200.

ALVARENGA, K. G.; G. C. Garcia, et al. (2007), "Epilepsia refratária: a experiência do Núcleo Avançado de Tratamento das Epilepsias do Hospital Felício Rocho (NATE) no período de março de 2003 a dezembro de 2006", Journal of Epilepsy and Clinical Neurophysiology, 13 (2), pp. 71-74.

BLAKELY, R. (2014), "Our Daughter, the Cannabis User", The Times. Disponível em: http://www.thetimes. co.uk/article/our-daughter-the-cannabis-user-m783zj3vp05.

BONDÍA, J. L. (2002), "Notas sobre a experiência e o saber de experiência", Revista Brasileira de Educação, (19), pp. 20-28.

BRASIL (1998), "Aprova o regulamento técnico sobre substâncias e medicamentos sujeitos a controle especial", Portaria n. ${ }^{\circ} 344$ da Secretaria de Vigilância Sanitária do Ministério da Saúde, Diário Oficial da União, Brasília-DF, Imprensa Nacional.

BRASIL (2000), "Atualiza as listas de substâncias entorpecentes, psicotrópicas, precursoras e outras sob controle especial, da Portaria SVS/MS n. ${ }^{\circ} 344^{\prime \prime}$, Resolução n 98 da Agência Nacional de Vigilância Sanitária, Diário Oficial da União, Brasília-DF, Imprensa Nacional.

BRASIL (2013a), "Altera a Resolução de Diretoria Colegiada - RDC 11, de 06 de março de 2013 que dispõe sobre a importação de substâncias sujeitas a controle especial e dos medicamentos que as contenham, e dá outras providências", Resolução n. 055 de 12 de dezembro de 2013 da Agência Nacional de Vigilância Sanitária, Diário Oficial da União, Brasília-DF, Imprensa Nacional.

BRASIL (2013b), "Aprova o regulamento para os programas de acesso expandido, uso compassivo e fornecimento de medicamento pós-estudo", Resolução n. ${ }^{\circ} 38$ da Agência Nacional de Vigilância Sanitária, Diário Oficial da União, Brasília-DF, Imprensa Nacional.

BRASIL (2014), "Aprova o uso compassivo do canabidiol para o tratamento de epilepsias da criança e do adolescente refratárias aos tratamentos convencionais", Resolução n. ${ }^{\circ} 2.113$ do Conselho Federal de Medicina, Diário Oficial da União, Brasília-DF, Imprensa Nacional.

BRASIL (2015a), "Dispõe sobre a atualização do Anexo I, Listas de substâncias entorpecentes, psicotrópicas, precursoras e outras sob controle especial, da Portaria SVS/MS n. 0 344/1998 e dá outras providências", Resolução n. ${ }^{\circ} 03$ da Agência Nacional de Vigilância Sanitária, Diário Oficial da União, Brasília-DF, Imprensa Nacional.

BRASIL (2015b), "Define os critérios e os procedimentos para a importação, em caráter de excepcionalidade, de produto à base de canabidiol em associação com outros canabinóides, por pessoa física, para uso próprio, mediante prescrição de profissional legalmente habilitado, para tratamento de saúde", Resolução n. ${ }^{\circ} 17$ da Agência Nacional de Vigilância Sanitária, Diário Oficial da União, Brasília-DF, Imprensa Nacional.

BRASIL (2016), "Dispõe sobre a atualização do Anexo I (Listas de substâncias entorpecentes, psicotrópicas, precursoras e outras sob controle especial) da Portaria SVS/MS n. ${ }^{\circ}$ 344", Resolução n. ${ }^{\circ} 66$ da Agência Nacional de Vigilância Sanitária, Diário Oficial da União, Brasília-DF, Imprensa Nacional.

BRASIL (2017), "Esclarecimentos a respeito do registro do medicamento Mevatyl", Nota Técnica n. ${ }^{\circ} 1$ da Agência Nacional de Vigilância Sanitária, Diário Oficial da União, Brasília-DF, Imprensa Nacional.

CARVALHO, V. M. e A. P. Martínez (2014), "Usos legales de cannabis y sus derivados en Latinoamérica", in J. T. Mosquera (org.), Marihuana cannabis: aspectos toxicológicos, clínicos, sociales y potenciales usos terapéuticos, Bogotá D. C./Colombia, Ministerio de Justicia e del Derecho.

CECCIM, R. B. e A. A. Ferla (2009), "Educação e saúde: ensino e cidadania como travessia de fronteiras", Trab. Educ. Saúde, 6 (3), pp. 443-456.

CECCIM, R. B. e L. C. M. Feuerwerker (2004), "Mudança na graduação das profissões de saúde sob o eixo da integralidade", Cadernos de Saúde Pública, 20 (5), pp. 1400-1410.

CORRÊA, M. P. (1926), Dicionário das plantas úteis do Brasil e das exóticas cultivadas, Rio de Janeiro, Imprensa Nacional, pp. 471-474.

CUNHA, J. M.; E. A. Carlini, et al. (1980), "Chronic Administration of Cannabidiol to Healthy Volunteers and Epileptic Patients", Pharmacology, 21 (3), pp. 175-185

ELSOHLY, M. A., et al. (2016), "Changes in Cannabis Potency Over the Last 2 Decades (1995-2014): Analysis of Current Data in the United States", Society of Biological Psychiatry, 79 (7), pp. 613-619.

GAONI, Y. e R. Mechoulam (1964), "Isolation, Structure, and Partial Synthesis of an Active Constituent of Hashish", Journal of the American Chemical Society, 86 (8), pp. 1646-1647.

HONÓRIO, K. M.; A. Arroio, et al. (2006), "Aspectos terapêuticos de compostos da planta Cannabis sativa", Química Nova, 29 (2), pp. 318-325.

HUSSAIN, S. A.; R. Zhou, et al. (2015), "Perceived Efficacy of Cannabidiol-Enriched Cannabis Extracts for Treatment of Pediatric Epilepsy: A Potential Role for Infantile Spasms and Lennox-Gastaut Syndrome", Epilepsy \& Behavior, (47), pp. 138-141.

KARNIOL, I. G. e E. A. Carlini (1972), "The Content of (-) $\Delta^{9}$-trans-tetrahydrocannabinol ( $\Delta^{9}$-thc) Does 
Not Explain All Biological Activity of Some Brazilian Marihuana Samples", J. Pharm. Pharmacol., 24 (10), pp. 833-834.

KARNIOL, I. G. e E. A. Carlini (1978), "Pharmacological Interaction Between Cannabidiol and $\Delta^{9}$-tetrahydrocannabinol", Psychopharmacology, 33 (1), pp. 53-70.

KARNIOL, I. G.; I. Shirakawa, et al. (1974), "Cannabidiol Interferes with the Effects of $\Delta^{9}$-tetrahydrocannabinol in Man", Eur. Journal of Pharmac., 28 (1), pp. 172-177.

KWAN, P. and M. J. Brodie (2000), "Early Identification of Refractory Epilepsy", New England Journal of Medicine, 342 (5), pp. 314-319.

MARANHÃO, M. V. M. e E. A. Gomes (2011), "Epilepsia e anestesia", Rev. Bras. Anestesiol, 61 (2), pp. 232-254.

McNAMARA, J. O. (2012), "Farmacoterapia das epilepsias", in L. L. Brunton, J. S. Lazo e K. L. Parker (orgs.), Goodman \& Gilman: as bases farmacológicas da terapêutica, Rio de Janeiro, McGraw-Hill Interamericana do Brasil.

MECHOULAM, R.; M. Peters, et al. (2007), "Cannabidiol - Recent Advances", Chem. Biodivers. , 4 (8), pp. 1678-1692.
MINISTÉRIO PÚBLICO FEDERAL (2016), Procuradoria da República do Distrito Federal. Justiça reitera decisão que libera tratamento com THC no Brasil. Disponível em: http://www.mpf.mp.br/df/sala-de-imprensa/noticias-df/justica-reitera-decisao-que-libera-tratamento-com-thc-no-brasil

NASCIMENTO, I. R.; H. B. Costa, et al. (2015), "Chemical Identification of Cannabinoids in Street Marijuana Samples Using Electrospray Ionization FT-ICR Mass Spectrometry", Analytical Methods, (4), pp. 1415-1424.

SERRES, M. (1993), Filosofia mestiça: le tiers-instruit, Rio de Janeiro, Nova Fronteira.

SILVA, R. A. D. (1929), Pharmacopeia dos Estados Unidos do Brasil, Rio de Janeiro, Companhia Editora Nacional, pp. 160-161.

TAKAHASHI, R. N.; A. W. Zuardi, et al. (1977), "Composição química e importância dos diversos constituintes na atividade farmacológica de amostras de cannabis sativa brasileira", Rev. Bras. de Pesquisas Méd. e Biol., 10 (6), pp. 379-385.

WHITING, P. F.; R. F. Wolff, et al. (2015), "Cannabinoids for Medical Use. A Systematic Review and Meta-analysis", Jama, 313 (24), pp. 2456-2473.

Recebido a 31/03/2017. Aceite para publicação a 24/10/2017.

Virgínia Martins Carvalho (virginiamc@pharma.ufrj.br). Universidade Federal do Rio de Janeiro, Faculdade de Farmácia, Departamento de Análises Clínicas e Toxicológicas, Laboratório de Análises Toxicológicas. Laboratório de Análises Toxicológicas, Av. Carlos Chagas Filho, 373, Bloco A, $2 .^{\circ}$ andar, Sala 3, 21941-902 Rio de Janeiro-RJ, Brasil.

Margarete Santos de Brito (contato@apepi.org). APEPI - Apoio à Pesquisa e Pacientes de Cannabis Medicinal. Laboratório de Análises Toxicológicas, Av. Carlos Chagas Filho, 373, Bloco A, $2^{\circ}$ andar, Sala 3, 21941-902 Rio de Janeiro-RJ, Brasil.

Mário Gandra (mgandra@ufrj.br). Universidade Federal do Rio de Janeiro, Faculdade de Farmácia, Departamento de Análises Clínicas e Toxicológicas. Laboratório de Desenvolvimento de Estratégias Educacionais (LDE²), Sala A2-05, Centro de Ciências da Saúde, Av. Carlos Chagas Filho, 373, 21941-902 Rio de Janeiro-RJ, Brasil. 